\title{
The importance of ultrasound examination in early arthritis
}

\author{
Monika Ponikowska ${ }^{1}$, Jerzy Świerkot ${ }^{2}$, Beata Nowak ${ }^{3}$ \\ ${ }^{1}$ Department of Rheumatology and Internal Medicine, Wrocław University Hospital, Wrocław, Poland \\ 2Department and Clinic of Rheumatology and Internal Medicine, Wrocław Medical University, Wrocław, Poland \\ ${ }^{3}$ Department of Pharmacology, Wrocław Medical University, Wrocław, Poland
}

\begin{abstract}
Objectives: To assess the importance of ultrasound (US) examination of joints in hands and feet in patients with early arthritis and perform comparative analysis of the diagnostic value of US examination for 8,12 and 52 selected joints.

Material and methods: 123 patients ( 87 women, 36 men) with arthritis lasting less than 12 months, naive to disease-modifying anti-rheumatic drugs and glucocorticosteroids. Necessary differential diagnostics was performed for each patient. After the preliminary analysis, 72 patients met the classification criteria for rheumatoid arthritis (RA) according to ACR/EULAR of 2010, and undifferentiated arthritis (UA) was diagnosed in 51 patients. UA patients were followed up after 6 and 12 months, and verification of the initial diagnosis yielded the following groups of patients: patients meeting classification criteria for RA, patients with maintained diagnosis of UA, patients in remission, and patients with other diagnoses. Ultrasound examination was performed considering the volume of joint effusion (JE), synovial membrane hypertrophy (GS), and synovial membrane hyperaemia assessed by power Doppler (PD). Results were assessed using the semi-qualitative scale. Coefficients being the sum of US scores for the assessment of JE, GS and PD for 52 and 12 joints in hands and feet, and 8 joints in hands were determined for the purpose of the study.

Results: In patients meeting classification criteria for RA during the initial assessment the US examination yielded significantly higher PD-52I, PD-12I and PD-8I coefficients. In UA patients who were diagnosed with RA after 12 months, the GS-8I coefficient was significantly higher.

Conclusions: Ultrasonography is a valuable tool in diagnostics of early arthritis. The GS assessment has prognostic value for UA patients. The assessment of 8 or 12 selected joints is often sufficient for the diagnostics of patients with early arthritis.
\end{abstract}

Key words: ultrasonography, early rheumatoid arthritis, synovitis.

\section{Introduction}

Early arthritis is a group of conditions referred to as unclassified arthritis, manifested by inflammation of a single or numerous joints, usually lasting for up to 12 weeks, or according to other authors, up to as long as 12 months [1]. Despite presence of inflammation of a joint, in the face of absence of typical symptoms, patients cannot be classified into any of the well-defined classes of inflammatory arthropathies. In approximately 20-60\% of patients the course of the disease may be self-lim- iting. Rheumatoid arthritis (RA) develops in 13-54\% of patients, and in other cases the particular disease may be diagnosed later [2-5]. The disease may also demonstrate no progression towards any of the typical arthropathies, and remain at the stage of undifferentiated inflammation.

Selection of patients at high risk of development of RA is particularly important, as they require early introduction of the modifying treatment. In clinical practice, ACR/EULAR classification criteria of 2010 are helpful in determination of the diagnosis of RA. Early diagnosis

\footnotetext{
Address for correspondence:

Monika Ponikowska, Department of Rheumatology and Internal Medicine, Wrocław University Hospital, 213 Borowska St., 50-556 Wrocław, Poland, e-mail: monika@proline.pl

Submitted: 30.11.2018; Accepted: 5.12.2018
} 
of RA is of key importance for introduction of an appropriate therapy. It is currently believed that introduction of disease-modifying anti-rheumatic drugs (DMARDs) within the first 3 months from development of symptoms (called the window of opportunity) is associated with much greater chance for remission, or at least low activity of the disease.

Our diagnostic options are expanded with imaging diagnostics, which should be used as a supplementary measure to the physical examination. Ultrasound (US) diagnostics is important, as it may supply some additional data regarding development of RA at its early stage, and activity of the disease $[6,7]$. The method allows visualisation of inflammation of the synovial membrane and erosions at the very early stage of the disease (discovery of synovial membrane hypertrophy [GS] is much more common with US, compared to the clinical examination). Similarly to magnetic resonance (MRI), US is particularly helpful in the assessment of arthritis, when lesions are too indistinct to be seen in radiographic examination [8]. Benefits associated with US involve a possibility to assess multiple joints at the same time, non-invasive character of the procedure, its broad availability and repeatability of the examination $[9,10]$.

The purpose of this study was to analyse differences occurring in the US image during the first examination of patients with early arthritis. We were interested in differences between those patients who were diagnosed with RA already at the initial visit, and those who were diagnosed with undifferentiated arthritis (UA) at the same visit, as well as the existence of any possible differences in the US image of patients depending on further evolution of their diagnosis, with a particular focus on the group of patients who developed RA in course of the further observation. Separation of prognostic factors in the situation of diagnostic doubts may bring great benefits to patients at risk of this destructive arthropathy.

\section{Material and methods Patients}

The 123 patients ( 87 women, 36 men) diagnosed in the Rheumatology Teaching Department between 2015 and 2017, with early arthritis were enrolled in the study. Inclusion criteria were: duration of symptoms up to 12 months, and oedema of at least 1 joint. Exclusion criteria included previous use of DMARDs or glucocorticosteroids. The mean age of patients at the initial visit was $49 \pm 17.7$ (18-80 years). The mean duration of symptoms from the initial examination was $5.9 \pm 3.9$ (1-12 months).

Patients $(n=72)$ met the ACR/EULAR classification criteria of 2010 for RA at the initial visit; 51 patients could not be diagnosed with any defined arthropathy, and were left with the diagnosis of UA. Patients with UA diagnosed initially were re-evaluated after 6 months and after 12 months.

The initial visit involved US examination of the hands and feet, and drawing blood for laboratory tests. The following were determined in all subjects: erythrocyte sedimentation rate (ESR), C-reactive protein level (CRP), rheumatoid factor level (RF) and the serum level of anti-citrullinated protein antibodies (ACPA). All patients were subjected to physical and clinical examination, and the Disease Activity Score 28 (DAS28) was calculated for them. The US examination was blinded for laboratory parameters.

All subjects gave their written informed consent for participation in the study. The project was positively assessed by the Bioethics Commission of Wrocław Medical University (no. 469/2010).

\section{Ultrasonography assessment}

Ultrasonography was performed in all subjects by the same physician possessing 10-year experience in US of the musculoskeletal system. Ultrasonography equipment was the MyLab25Gold (Esaote) with a linear head (frequency range: $12-18 \mathrm{MHz}$ ) used in all cases. The power Doppler (PD) signal was obtained following reduction of artefacts.

The following joints were examined: bilaterally wrist joints (2), metacarpophalangeal joints (MCP; 10), proximal interphalangeal joints (PIP; 8), distal interphalangeal joints (10), tarsal joints (2), metatarsophalangeal joints (MTP; 10), interphalangeal joint of the great toe (2), and proximal interphalangeal joints of toes (8). In total 52 joints were assessed in each patient.

The US assessed: volume of joint effusion, GS, activity of inflammation in the PD examination.

The volume of joint effusion (JE), the assessment of GS, and activity of inflammation in PD were graded according to the 4-grade semi-quantitative scale proposed by Szkudlarek [11].

Joint effusion was defined as abnormal, usually echoless, intraarticular, compressible material: 0 - normal volume of articular fluid, 1 - minor increase of the volume of the articular fluid, 2 - moderate increase of the volume of the articular fluid, 3 - major increase of the volume of the articular fluid.

Joint effusion 52-Index (JE-52I) - for each patient the index was calculated as the sum of grades obtained for presence of effusion in all 52 joints.

Joint effusion 8-Index (JE-8I) - the sum of grades for the presence of effusion in 8 joints (bilateral wrist joints, proximal interphalangeal joints II, III, V)

Joint effusion 12-Index (JE-12I) - the sum of grades for the presence of effusion in 12 joints (bilateral wrist 
joints, proximal interphalangeal joints II, III, V and MTP II and V).

Synovial hypertrophy (synovitis) - it is an abnormal, hypoechogenic, intraarticular, hardly compressible tissue, usually assessed on the grey scale: 0 - no synovial hypertrophy, 1 - minor synovial hypertrophy only in physiological spaces of the joint, 2 - moderate synovial hypertrophy with elevation of the articular capsule, 3 - major synovial hypertrophy forming numerous pathological recesses of the articular capsule.

Synovial hypertrophy 52-Index (GS-52I) - for each patient the index was calculated as the sum of grades obtained for intensity of synovial hypertrophy in 52 joints.

Synovial hypertrophy 8-Index (GS-8I) - the sum of grades for intensity of synovial hypertrophy in 8 joints (bilateral wrist joints, proximal interphalangeal joints II, III, V).

Synovial hypertrophy 12-Index (GS-12I) - the sum of grades for intensity of synovial hypertrophy in 12 joints (bilateral wrist joints, proximal interphalangeal joints II, III, V, and MTP II and V).

The PD signal is defined as presence of signals of vascular flow within the hypertrophic synovial membrane: 0 - no vascular signals, 1 - single vascular signals, 2 - present vascular signals occupy less than $50 \%$ of the surface of hypertrophic synovial membrane, 3 - diffuse vascular signals visible over more than $50 \%$ of the surface of hypertrophic synovial membrane.

Power Doppler 52-Index (PD-52I) - for each patient the index was calculated as the sum of grades obtained for hyperaemia of the synovial membrane in 52 joints.

Power Doppler 8-Index (PD-8I) - the sum of grades for hyperaemia of the synovial membrane in 8 joints (bilateral wrist joints, proximal interphalangeal joints II, III, V).

Power Doppler 12-Index (PD-12I) - the sum of grades for hyperaemia of the synovial membrane in 12 joints (bilateral wrist joints, proximal interphalangeal joints II, III, V, MTP II and V).

Joint effusion intensity, synovial hypertrophy and PD activity of inflammation were compared to laboratory parameters: ESR, CRP, RF and ACPA, assessed on a 2-grade scale:

- ESR: 0 - correct value (3-15 mm for women, 1-10 mm for men), 1 - increased value (over $15 \mathrm{~mm}$ for women, over $10 \mathrm{~mm}$ for men).

- CRP: 0 - normal level (0-5 mg/l), 1 - increased level (over $5 \mathrm{mg} / \mathrm{l}$ ).

- RF: 0 - normal titre (0-14 IU/ml), 1 - increased titre (over $14 \mathrm{IU} / \mathrm{ml}$ ).

- ACPA: 0 - absent antibodies (less than $5.0 \mathrm{U} / \mathrm{ml}$ ), 1 - present antibodies (over $5.0 \mathrm{U} / \mathrm{ml}$ ).

\section{Statistical analysis}

Statistical analysis was performed using Statistica 12. Statistical tests used in the study included Student's t-test and ANOVA with NIR post hoc test. In order to evaluate possible correlations Spearman's rank correlation coefficient and Pearson's correlation coefficient were used. The threshold set for statistical significance was $p<0.05$.

\section{Results}

Based on the initial examination, RA was diagnosed in 72 patients, and UA in the remaining 51. Table I presents characteristics of both groups. Based on those results it was found that RA patients had significantly more often elementary education (48.6\%), but no differences in terms of sex, family history or smoking history were found. Presence of RF (75\%) and ACPA (76.4\%) were significantly more often found in RA patients.

Additionally, RA patients demonstrated a significantly higher number of painful and oedematous joints in physical examination, and a higher value of the DAS28 index. Ultrasonography of RA patients indicated a higher value of the effusion index for 52 and 12 joints, and a higher value of the index of synovial hyperaemia for 52, 12 and 8 joints.

In all patients with early arthritis, the DAS28 value was correlated with all parameters assessed in US, and PD-521, PD-8I and PD-12I were additionally correlated with ESR and CRP. Correlations of the above-mentioned parameters are presented in Table II.

Patients with diagnosed UA were further observed. Six and 12 months later their diagnoses were verified, and the following groups were obtained as a final result: patients meeting classification criteria for RA, patients with maintained diagnosis of UA, patients in remission, and patients with other diagnoses (psoriatic arthritis -2, seronegative spondyloarthropathy -2 , reactive arthritis -4 , Sjögren syndrome -1 , remitting seronegative symmetrical synovitis with pitting oedema -2 , tumours -1 , polymyalgia rheumatica - 1 , systemic lupus erythematosus - 1 , mixed disease of connective tissue - 1, undifferentiated systemic disease of connective tissue - 1). One patient failed to report at a control visit, and 50 patients were further analysed. Results are presented in Table III.

Individual groups were significantly different in terms of body weight, BMI and ESR. Additionally, a statistically significant difference was present in the number of painful and oedematous joints, and in US in values of the index of synovial hypertrophy for 8 joints, and in values of indexes of synovial hyperaemia for 8 and 12 joints. 
Table I. Demographic, clinical, laboratory, and ultrasound characteristics of patients

\begin{tabular}{|c|c|c|c|}
\hline Parameters & $\begin{array}{c}\text { RA } \\
(n=72)\end{array}$ & $\begin{array}{c}\text { UA } \\
(n=51)\end{array}$ & $p$-value \\
\hline Age $^{*}$ & $51.3 \pm 16(18-80)$ & $46.5 \pm 19(18-85)$ & NS \\
\hline Female gender ${ }^{\star \star}$ & $50(69.5)$ & $37(72.5)$ & NS \\
\hline Weight* & $71.7 \pm 14.8(50-120)$ & $69.7 \pm 14.5(49-110)$ & NS \\
\hline$B M 1^{*}$ & $25 \pm 4.4(19-42)$ & $25 \pm 4.8(17-35)$ & NS \\
\hline $\operatorname{ESR}(\mathrm{mm} / \mathrm{h})^{\star}$ & $39.9 \pm 32(5-125)$ & $34.7 \pm 30(3-105)$ & NS \\
\hline $\operatorname{CRP}(\mathrm{mg} / \mathrm{l})^{*}$ & $29.7 \pm 39.8(4-179)$ & $30.5 \pm 53.1(0.08-238)$ & NS \\
\hline RF positive ${ }^{\star *}$ & $54(75)$ & $8(15.7)$ & $<0.001$ \\
\hline RF positive high titre ${ }^{* *}$ & $36(50)$ & $3(5.9)$ & $<0.001$ \\
\hline ACPA positive ${ }^{* *}$ & $55(76.4)$ & $2(3.9)$ & $<0.001$ \\
\hline ACPA positive high titre ${ }^{\star *}$ & $50(69.4)$ & $2(3.9)$ & $<0.001$ \\
\hline Smoker** & $32(44.4)$ & $18(25)$ & NS \\
\hline Positive family history for $R A^{* *}$ & $11(15.3)$ & $8(15.7)$ & NS \\
\hline$N P J^{*}$ & $9.1 \pm 4.4(2-19)$ & $5.9 \pm 4.4(0-18)$ & $<0.001$ \\
\hline NSJ $J^{*}$ & $8.4 \pm 4.3(0-18)$ & $6 \pm 4(0-17)$ & $<0.05$ \\
\hline VAS* $^{*}$ & $61.9 \pm 20.2(12-100)$ & $55.1 \pm 20.2(5-90)$ & NS \\
\hline DAS28* & $5.6 \pm 1.1(3-8.3)$ & $4.8 \pm 1.3(2.1-7.6)$ & $<0.001$ \\
\hline$J E-521^{*}$ & $34.3 \pm 16.3(8-88)$ & $28.7 \pm 12.6(7-64)$ & $<0.05$ \\
\hline$J \mathrm{E}-81^{\star}$ & $10.9 \pm 4.4(2-23)$ & $9.5 \pm 4.9(1-18)$ & NS \\
\hline$J E-\left.12\right|^{\star}$ & $15.8 \pm 5.5(4-31)$ & $13.7 \pm 5.8(4-25)$ & $<0.05$ \\
\hline GS-521* & $16 \pm 11(0-58)$ & $13 \pm 9.6(3-40)$ & NS \\
\hline GS-81* & $6.2 \pm 4.4(0-16)$ & $5.2 \pm 4.5(0-16)$ & NS \\
\hline GS-12/* & $8.6 \pm 5.4(0-21)$ & $7.3 \pm 5.6(0-20)$ & NS \\
\hline PD-521* & $7.7 \pm 7.8(0-47)$ & $2.8 \pm 2.9(0-14)$ & $<0.001$ \\
\hline PD-81* & $4.5 \pm 3.9(0-17)$ & $1.9 \pm 2(0-6)$ & $<0.001$ \\
\hline PD-121* & $5.2 \pm 4.5(0-19)$ & $2 \pm 2(0-6)$ & $<0.001$ \\
\hline
\end{tabular}

${ }^{*}$ Results are presented as mean \pm standard deviation (range). ${ }^{* *}$ Results are presented as number of patients (\%). RA - rheumatoid arthritis; UA - undifferentiated arthritis; BMI - body mass index; ESR - erythrocyte sedimentation rate; CRP - C-reactive protein; $R F$ - rheumatoid factor; ACPA - anti-citrullinated protein antibodies; NPJ - number of painful joints; NSJ - number of swollen joints; VAS - visual analogue scale; DAS28 - Disease Activity Score 28; JE-52I - Joint effusion 52-Index; JE-81 - Joint effusion 8-Index; JE-12I - Joint effusion 12-Index; GS-521 - Synovial hypertrophy 52-Index; GS-81 - Synovial hypertrophy 8-Index; GS-121 - Synovial hypertrophy 12-Index; PD-521 - power Doppler 52-Index; PD-81 - power Doppler 8-Index; PD-121 - power Doppler 12-Index.

Body weight and BMI were significantly higher in patients with UA compared to groups of patients with other diagnoses and in remission. No statistically significant differences of those parameters were found between RA patients and other groups.

The mean ESR value was significantly higher in the group of patients with other diagnoses, compared to patients in remission and UA patients. No statistically significant differences were found between RA patients and other groups.

The number of painful and oedematous joints and the synovial hypertrophy index for 8 joints were significantly higher in RA patients, compared to all other groups.
The index of synovial hyperaemia for 8 and 12 joints was significantly higher in RA patients, compared to groups of patients in remission and with other diagnoses. No statistically significant differences were found between UA patients and other groups.

\section{Discussion}

In this study, 1/4 of patients met criteria of RA after 12 months, and $1 / 3$ were still diagnosed with UA. In 14\% of patients arthritis was of a self-limiting character. The remaining group of patients evolved towards other particular rheumatological diseases.

Based on the present study it was demonstrated that some significant differences in US were found 
Table II. Correlations between disease activity and results of undifferentiated arthritis of patients with early arthritis

\begin{tabular}{|lccccccccc|}
\hline Variable & JE-52I & JE-12I & JE-8I & GS-52I & GS-12I & GS-8I & PD-52I & PD-12I & PD-8I \\
\hline ESR $(\mathrm{mm} / \mathrm{h})$ & 0.0422 & 0.0303 & 0.0803 & 0.0403 & 0.0106 & 0.0521 & $0.3098^{\star}$ & $0.3010^{*}$ & $0.3106^{\star}$ \\
\hline CRP $(\mathrm{mg} / \mathrm{l})$ & 0.0845 & 0.0537 & 0.1220 & 0.0489 & 0.0089 & 0.0619 & $0.3528^{\star}$ & $0.3618^{*}$ & $0.3963^{\star}$ \\
\hline DAS28 & $0.3467^{\star}$ & $0.3832^{\star}$ & $0.4435^{\star}$ & $0.2856^{\star}$ & $0.3087^{\star}$ & $0.3667^{\star}$ & $0.4330^{\star}$ & $0.5129^{\star}$ & $0.5261^{\star}$ \\
\hline
\end{tabular}

ESR - erythrocyte sedimentation rate; CRP - C-reactive protein; DAS28 - Disease Activity Score 28; "correlation coefficients are significant at $p<0.05$

Table III. Laboratory results and results of ultrasound examination of patients with initial diagnosis of undifferentiated arthritis

\begin{tabular}{|c|c|c|c|c|c|}
\hline Parameters & RA & UA & Other diagnoses & Remission & $\begin{array}{c}\text { ANOVA with post hoc } \\
\text { NIR test } \\
p<0.05\end{array}$ \\
\hline Number of patients & 12 & 15 & 16 & 7 & \\
\hline Age & $52.2 \pm 17.6$ & $48 \pm 18$ & $43.7 \pm 21.6$ & $40.9 \pm 20.5$ & NS \\
\hline Weight & $69.8 \pm 14.5$ & $78.1 \pm 15.5$ & $62.8 \pm 8.4$ & $63.9 \pm 13.3$ & $\cdots$, ооо \\
\hline BMI & $25.5 \pm 3.9$ & $28.2 \pm 4.9$ & $22.6 \pm 3.5$ & $24 \pm 5.8$ & $\cdots$, ооо \\
\hline $\operatorname{ESR}(\mathrm{mm} / \mathrm{h})$ & $33.5 \pm 30$ & $28.1 \pm 32.7$ & $47.9 \pm 28.5$ & $20.4 \pm 24.2$ & ${ }^{x \times x}, \cdots$ \\
\hline CRP $(\mathrm{mg} / \mathrm{l})$ & $24.3 \pm 38.7$ & $31.6 \pm 64.5$ & $44.6 \pm 62.2$ & $7.9 \pm 12.4$ & NS \\
\hline NPJ & $9.3 \pm 4.8$ & $5.3 \pm 4.4$ & $4 \pm 3.1$ & $5.1 \pm 3$ & ${ }^{* \star \star},+++, \# \#$ \\
\hline NSJ & $9.3 \pm 3.6$ & $5 \pm 3.9$ & $4.8 \pm 3.4$ & $5.1 \pm 3$ & ${ }^{\star \star \star},+++, \# \#$ \\
\hline VAS & $59 \pm 19$ & $58.5 \pm 23$ & $55.7 \pm 19.1$ & $40.7 \pm 17.4$ & NS \\
\hline DAS28 & $5.5 \pm 1.3$ & $4.6 \pm 1.4$ & $4.8 \pm 1.1$ & $4.2 \pm 1$ & NS \\
\hline JE-52I & $29.5 \pm 11.1$ & $27.3 \pm 14$ & $28.3 \pm 12.2$ & $27.3 \pm 14$ & NS \\
\hline JE-8I & $12.4 \pm 3.8$ & $9 \pm 5$ & $7.9 \pm 4.6$ & $7.9 \pm 5.5$ & NS \\
\hline$J E-12 \mid$ & $16.8 \pm 4.3$ & $13 \pm 6.2$ & $12.3 \pm 5.3$ & $11.6 \pm 6.9$ & NS \\
\hline GS-52I & $14 \pm 10.3$ & $12.7 \pm 10.2$ & $11.8 \pm 7.8$ & $11 \pm 9.4(6-32)$ & NS \\
\hline GS-8I & $8.4 \pm 5.4$ & $4.5 \pm 3.6$ & $3.9 \pm 3.5$ & $3.2 \pm 4.3$ & ${ }^{* \star \star},{ }^{+++}, \# \# \#$ \\
\hline GS-12I & $10.3 \pm 6.7$ & $6.9 \pm 4.9$ & $5.7 \pm 4.4$ & $5.4 \pm 5.7$ & NS \\
\hline PD-521 & $3.7 \pm 2.8$ & $2.7 \pm 2.8$ & $2.8 \pm 3.5$ & $1.7 \pm 1.5$ & NS \\
\hline PD-8I & $3.3 \pm 2.4$ & $2 \pm 1.9$ & $1.3 \pm 1.7$ & $0.4 \pm 0.8$ & ${ }^{+++}, \# \#$ \\
\hline PD-12I & $3.4 \pm 2.4$ & $2 \pm 1.9$ & $1.7 \pm 1.8$ & $0.4 \pm 0.8$ & +++, \#\#\# \\
\hline
\end{tabular}

Results are presented as mean \pm standard deviation (range). RA - rheumatoid arthritis; UA - undifferentiated arthritis; ${ }^{* * *} p<0.05 R A$ vs. UA; ${ }^{+++} p<0.05$ RA vs. other diagnoses; ${ }^{\# \#} p<0.05$ RA vs. remission; $\cdots p<0.05$ UA vs. other diagnoses; ${ }^{\circ 00} p<0.05$ UA vs. remission;

${ }^{\times \times \times} p<0.05$ other diagnoses vs. remission.

between patients meeting classification criteria of RA during their initial visit, and those diagnosed with UA at that visit, besides statistically significant differences in the clinical assessment, such as the number of painful and oedematous joints, and DAS28. Those differences regarded both the assessment of the volume joint effluence, and intensity of synovial hyperaemia. It should also be stressed that data were similar for the assessment of 52 joints, and for 12 selected joints of the hands and feet, and also for the PD assessment of just 8 joints in the hands. It is an important argument in favour of performing US examination of a lower number of joints, most commonly affected in RA. That leads to a significantly shorter time needed for the procedure, with no impact on its diagnostic value.

Sun et al. [12] presented a similar simplified system of US assessment, regarding selected hand joints in RA patients. Eight joints were assessed in some of the patients (bilateral wrist joints, MCP II, III, V), and 22 joints in the rest of the group. The researchers presented US assessment consistent with that presented by the present authors, using the semi-quantitative scale for GS and PD. Determined indexes for GS and PD for 8 joints were well correlated with the GS and PD index for 22 joints. 
In their conclusions Sun et al. recommended a clinical practice of shortened US for the assessment of synovitis in RA patients.

The selection of joints to be preferably examined with US was also analysed by Yoshimi et al. [13]. The authors assessed the usefulness of the US examination depending on the selection of examined joints. 406 diagnosed with RA were assessed. In that study US was used for the assessment of activity of the disease. Eight joints were routinely examined (wrists, MCP II, III and knees) in all patients. In some of the patients, besides the selected 8 joints, also some additional joints were examined, identified by patients themselves as the most symptomatic. Sensitivity and specificity of the US assessment of RA activity for 8 selected joints were significantly higher than the assessment of additional joints appointed by patients. The assessment of additional joints suggested by patients demonstrated synovitis in $38 \%$ of cases, despite absence of changes in the typical 8 joints. It was concluded that the assessment of additional joints was significant, but not sufficient for the monitoring of RA. Summing up, the researchers stated that the most appropriate procedure for the assessment of activity of the disease involved US of joints suggested by patients, along with routinely assessed joints. In our study we did not consider the opinions of patients. However, as it was demonstrated in the present study, examination of joints typically involved in RA is an appropriate procedure also in the case of diagnostics of patients with early arthritis.

Advantages associated with US were also presented by Mueller et al. [14]. The authors compared examination of hand joints in patients meeting ACR/EULAR criteria of 2010 for RA with manual US equipment, with automated US (ACUSON S2000 TM ABVS), MRI and clinical examination. Both US assessments were clearly superior to the clinical examination in terms of detection of oedematous joints. However, automated US was not sufficient for the assessment of synovitis. Additionally, currently the assessment of the PD option is currently unavailable using that method. PD assessment is necessary for the diagnosis of early arthritis. Authors point to necessary further improvement of the automated US method.

Based on detailed analysis of UA patients assessed in our study, statistically significantly higher values of the GS index were found for selected hand joints (GS-8I) in patients who were subsequently diagnosed with RA. No similar correlation was found for the assessment of PD. The reason may be a rather low mean CRP value in this group of patients. In our previous study we demonstrated that higher CRP values were correlated with higher values of the PD index [15].

A similar group was analysed by Alivernini et al. [16]. In their study focusing, among others, on the US as- sessment of patients with sero-negative UA that further evolved into arthritis meeting criteria for defined arthropathies (including RA) the authors also found higher values of PDUS (power Doppler ultrasound), besides higher values of GSUS (grey scale ultrasound). They assessed wrist joints, MCP II-III, knees, and MTP II-V. Besides differences in selection of joints, also the number of patients evolving to RA was lower in that study. In our study, a significantly higher baseline value for GS-8I was obtained only for patients with the final diagnosis of RA.

Salaffi et al. [17] examined 149 patients with UA. Twelve months later 62 patients with RA were isolated from that group, and the authors demonstrated that the presence of PD was a factor favouring progression of UA into RA. The study was performed using the analysis of US presentation of hand joints (wrists, MCP II-V, PIP II-V). $P D>1$ was regarded as positive. Summing up, the authors stated that RA occurred statistically significantly more commonly in the group of patients with PD $>1$ in at least 2 joints. The study did not analyse the GS presentation. We found no correlation between PDUS and the final diagnosis of RA. Differences in results obtained by the authors of this study may result from, among other factors, a significant difference in volumes of study groups.

In another paper by Freeston et al. [18] regarding UA patients with absent RF and ACPA, the authors underlined the role of GS $3, P D \geq 1$, and of at least one erosion, occurring together or independently in US. The researchers stressed the significant role of those lesions observed in US. Their presence significantly increased the probability of development of early rheumatoid arthritis.

The study presented by Stadt et al. [19] regarded the US assessment of patients with arthralgia (192 patients), with a positive RF and/or ACPA The authors presented the following lesions observed in US that constituted factors favouring the development of arthritis: $J E \geq 2, G S \geq 2$ and $P D \geq 1$. However, they demonstrated no statistical significance. In this study patients with existing clinical symptoms of arthritis were analysed.

The importance of the assessment of synovitis by GS was underlined by Ying-hua Zang et al. [20]. The researchers assessed 189 patients with non-specific ailments of the musculoskeletal system, and compared sensitivity and specificity of US, radiographic methods, and the clinical examination. GS was assessed in wrists, MCP II-III, and PIP II-III. US proved to be the most specific for the assessment of early synovitis. The authors underlined the role of the examination as a precise, cheap and readily available method.

The study by Ventura-Rios et al. [21] presented a different system of US assessment of joints than that presented in this paper. The system was presented by Outcome Measures in Rheumatoid Arthritis, Clinical Tri- 
als - European League Against Rheumatism (OMERACT-EULAR) and regarded the assessment of synovitis. It is known as GLOVES, and it is a combination of GS and PD. The scale offers the scoring range from 0 to 3 , while the score involves both the information on synovial hypertrophy and the PD signal. The study was aimed at the comparative US analysis using the semi-quantitative scale, separately for GS and PD, and the GLOVES scale in patients diagnosed with RA. Hand joints - wrists, MCP II-III, PIP II-III, MTP II and V - were assessed in each patient. Similar results were obtained using both scales. According to Ventura-Rios et al. the assessment of GS is more objective, because differences in the assessment of PD may to a greater extent result from differences between US equipment, and from various experience of a US operator. Based on our observations it was inferred that PD assessment was necessary already at the first stage of the path towards the final diagnosis of a patient with early arthritis, because the value of the PD index was significantly higher in the group of RA patients compared to UA patients. US of the musculoskeletal system requires experience, but associated diagnostic benefits are considerable.

Horton et al. [22] analysed a group of UA patients. The authors examined 26 joints (elbow, wrist, MCP and PIP II-III, knee, tarsal, and MTP I-V). They assessed the global sum of GS and PD in all joints, and the number of joints with $G S \geq 2$ and $P D \geq 1$. Additionally, they analysed presence of the following changes in US: GS $=3$ in MTP joints or GS $\geq 2$ in other joints, and $P D \geq 2$ in wrists, and MTP I and PD $\geq 1$ in other joints. Control observation performed 12 month later indicated that $22 \%$ of patients met ACR/EULAR criteria of 2010, and $53 \%$ of patients remained with the diagnosis of $U A$. The risk of progression to RA was associated with the increase of total GS. To conclude, the authors stated that determination of the presence of at least 2 joints assessed as GS $\geq 2$ of 26 examined joints determined a future application of methotrexate therapy, and that GS $\geq 2$ in at least 5 joints, or GS $\geq 3$ in MTP joints, and GS $\geq 2$ in other joints, significantly increased the risk of fully symptomatic RA. The results of the study confirmed the prognostic value of US, and particularly of the GS assessment in UA patients. The same was shown in our study.

The significance of US in patients diagnosed for RA (depending on the presence of the RF) was also assessed by Minowa et al. [23]. The researchers analysed patients with initial ailments of hand joints, diagnosed with RA in the further follow-up (52 of 122 patients). Twenty-two joints (wrists, MCP, PIP) were examined at baseline and after 24 weeks. Total GS/PD was significantly higher in RA patients. In the conclusion the authors stated that US was an examination method providing a significant input in the diagnosis of RA, and that the US presentation could be variable depending on the presence of immunological markers.

Barbulescu et al. [24] assessed the prognostic value of RA33, and its correlation with lesions assessed in US (hand joints: wrists, MCP II-V, PIP) in 29 patients with early rheumatoid arthritis. Ultrasonography was performed as part of the initial diagnostics, and 12 months later. The initial US demonstrated at least 1 joint with $\mathrm{GS} \geq 1$ (in all patients), $P D \geq 2$ in at least 1 joint in 23 patients, $P D \geq 2$ in at least 1 joint in 20 patients, and PD $\geq 3$ in at least 1 joint in 6 patients. It was concluded that besides its diagnostic value anti-RA33 could be important in identification of patients with a mild course of the disease, manifested by low activity of synovitis.

Limitations of our study included a low number of patients with UA and a short follow-up. The strength of the study was that we examined lower distal extremity joints.

\section{Conclusions}

Ultrasonography of the musculoskeletal system is a very important examination that facilitates the diagnosis, and plays an important predictive role in doubtful cases, as well as supporting the assessment of activity of the disease. The GS assessment has prognostic value for UA patients. The assessment of 8 or 12 selected joints is often sufficient for the diagnosis of patients with early arthritis. However, to be reliable it has to be performed by an experienced operator, using appropriate equipment.

The authors declare no conflict of interest.

\section{References}

1. Jeka S, Wojciechowski R. Wczesne zapalenie stawów. Reumatologia News 2016; 1: 61-66.

2. Norli ES, Brinkmann GH, Kvien TK, et al. Diagnostic spectrum and 2-year outcome in a cohort of patients with very early arthritis. RMD Open 2017; 3: e000573.

3. Hua C, Daien Cl, Combe B, et al. Diagnosis, prognosis and classification of early arthritis: results of a systematic review informing the 2016 update of the EULAR recommendations for the management of early arthritis. RMD Open 2017; 3: 1-10.

4. Söderlin MK, Börjesson O, Kautiainen $\mathrm{H}$, et al. Annual incidence of inflammatory joint diseases in a population based study in southern Sweden. Ann Rheum Dis 2002; 61: 911-915.

5. Hazes JMW, Luime JJ. The epidemiology of early inflammatory arthritis. Nat Rev Rheumatol 2011; 7: 381-390.

6. Jeka S, Zuchowski P, Dura M, et al. The role of ultrasonography in the diagnostic criteria for rheumatoid arthritis and monitoring its therapeutic efficacy. Adv Clin Exp Med 2018; 27: 1303-1307. 
7. Jeka S, Dura M, Żuchowski P, et al. The role of ultrasonography in monitoring long-standing rheumatoid arthritis: a pilot study. Reumatologia 2017; 55: 177-182.

8. Wakefield RJ, Gibbon WW, Conaghan PG, et al. The value of sonography in the detection of bone erosions in patients with rheumatoid arthritis: a comparison with conventional radiography. Arthritis Rheum 2000; 43: 2762-2770.

9. Kawashiri SY, Suzuki T, Okada A, et al. Musculoskeletal ultrasonography assists the diagnostic performance of the 2010 classification criteria for rheumatoid arthritis. Mod Rheumatol 2013; 23: 36-43.

10. Naredo E, Collado P, Cruz A, et al. Longitudinal power Doppler ultrasonographic assessment of joint inflammatory activity in early rheumatoid arthritis: Predictive value in disease activity and radiologic progression. Arthritis Rheum 2007; 57: 116-124.

11. Szkudlarek M, Court-Payen M, Jacobsen S, et al. Interobserver agreement in ultrasonography of the finger and toe joints in rheumatoid arthritis. Arthritis Rheum 2003; 48: 955-962.

12. Sun X, Deng X, Geng Y, et al. A simplified and validated ultrasound scoring system to evaluate synovitis of bilateral wrists and hands in patients with rheumatoid arthritis. Clin Rheumatol 2018; 37: 185-191.

13. Yoshimi R, Takeno M, Toyota Y, et al. On-demand ultrasonography assessment in the most symptomatic joint supports the 8-joint score system for management of rheumatoid arthritis patients. Mod Rheumatol 2017; 27: 257-265.

14. Mueller R, Grunke M, Wendler J, et al. The Value of an Automated Ultrasound System in the Detection of Synovitis. Ultrasound Int Open 2018; 4: E61-E68.

15. Ponikowska M, Wiland P. The initial ultrasonographic examination of hands and feet joints in patients with early rheumatoid arthritis. Reumatologia 2015; 53: 179-185.

16. Alivernini S, Tolusso B, Petricca L, et al. Synovial Predictors of Differentiation to Definite Arthritis in Patients With Seronegative Undifferentiated Peripheral Inflammatory Arthritis: microRNA Signature, Histological, and Ultrasound Features. Front Med (Lausanne) 2018; 5: 186.
17. Salaffi F, Ciapetti A, Gasparini S, et al. A clinical prediction rule combining routine assessment and power doppler ultrasonography for predicting progression to rheumatoid arthritis from early-onset undifferentiated arthritis. Clin Exp Rheumatol 2010; 28: 686-694.

18. Freeston JE, Wakefield RJ, Conaghan PG, et al. A diagnostic algorithm for persistence of very early inflammatory arthritis: The utility of power Doppler ultrasound when added to conventional assessment tools. Ann Rheum Dis 2010; 6: 417-419.

19. van de Stadt LA, Bos WH, Meursinge Reynders $M$, et al. The value of ultrasonography in predicting arthritis in auto-antibody positive arthralgia patients: A prospective cohort study. Arthritis Res Ther 2010; 12: R98.

20. Zhang Y-H, Li K, Xiao J, et al. Comparison of Ultrasound, Radiography, and Clinical Investigations in the Diagnosis of Early Rheumatoid Synovitis in Patients with Nonspecific Musculoskeletal Symptoms: A Multicenter Cross-Sectional Study. Med Sci Monit 2018; 24: 4372-4378.

21. Ventura-Ríos L, Hernández-Díaz C, Ferrusquia-Toríz D, et al. Reliability of ultrasound grading traditional score and new global OMERACT-EULAR score system (GLOESS): results from an inter- and intra-reading exercise by rheumatologists. Clin Rheumatol 2017; 36: 2799-2804.

22. Horton SC, Tan AL, Wakefield RJ, et al. Ultrasound-detectable grey scale synovitis predicts future fulfilment of the 2010 ACR/EULAR RA classification criteria in patients with new-onset undifferentiated arthritis. RMD Open 2017; 3: e000394.

23. Minowa K, Ogasawara M, Murayama G, et al. Predictive grade of ultrasound synovitis for diagnosing rheumatoid arthritis in clinical practice and the possible difference between patients with and without seropositivity. Mod Rheumatol 2016; 26 : 188-193.

24. Barbulescu AL, Ciurea PL, Mitran C, et al. High frequency ultrasonography of the hand versus anti-RA33 evaluation in early rheumatoid arthritis - A pilot study. Med Ultrason 2017; 19: 166-171. 